\title{
On the Formation and Stability of Two Misfit Dislocations in the $\mathrm{Cu}-\gamma \mathrm{Fe}$ System*
}

\author{
Arun KUMAR**, Monika GAUTAM** and Anandh SUBRAMANIAM** \\ ** Department of Material Science and Engineering, Indian Institute of Technology Kanpur, \\ Kanpur 208016, INDIA \\ E-mail: anandh@iitk.ac.in
}

\begin{abstract}
On growth beyond a critical size coherent precipitates become semicoherent by the formation of an interfacial misfit edge dislocation loop. On further growth of the precipitate, the interface progressively loses its coherency by the formation of additional dislocation loops. The current investigation pertains to the energetic feasibility of two dislocation loops. Finite element simulations are performed to simulate the stress state of the semicoherent precipitate and to compute the radius of the precipitate at which two symmetrically positioned dislocation loops become energetically favourable. Aspects regarding the stability of the loops are also explored using the simulations. $\mathrm{Cu}-\gamma \mathrm{Fe}$ system is used as a model system to illustrate the methodology and to capture the essential aspects of the process.
\end{abstract}

Key words: Semi-Coherent Precipitate, Critical Size, Finite Element Method

\section{Introduction}

Interfacial misfit dislocation loops can partially relax the coherency strains, on the growth of the precipitate beyond a critical size $\left(\mathrm{r}^{*}\right)[1,2]$. This implies that for a precipitate of size bigger than $r^{*}$, it is energetically favourable for the formation a dislocation loop (i.e. the semi-coherent interface is preferred over the coherent one). The value of $r^{*}$ for a spherical precipitate is given by [2-4]:

$$
r^{*}=\frac{b}{8 \pi \varepsilon(1-v)}\left[\ln \frac{8 r^{*}}{b}+\frac{3-2 v}{4(1-v)}-1\right]
$$

Where, ' $\varepsilon$ ' $\left(\varepsilon=\left[(1+v) f_{m}\right] /[3(1-v)][3,5]\right)$ is the strain parameter of a constrained system (i.e. of the precipitate), ' $f \mathrm{~m}$ ' is the lattice misfit between the precipitate and the matrix, ' $b$ ' is the modulus of the burgers vector and ' $v$ ' is the Poisson's ratio of the precipitate.

As the semicoherent precipitate with a single misfit dislocation loop grows, multiple loops can form at the interface, to further relieve the misfit strain. Brooks had developed a criterion for the existence of an array of equally spaced misfit dislocations [6]. The critical radius as computed by Brooks is given by: $r^{*} \approx b / 2 \delta$ (where, $\delta$ is the fractional difference in lattice parameters). Experimental investigations on the formation of multiple dislocation loops also exist in literature [7,8], along with theoretical work (addressing certain aspects of the process) [9]. The finite element method (FEM) has proved to be a useful tool in the study of both precipitation [10,11] and dislocations [12,13].

On one hand, there have been considerable investigations on the issue of critical size, while on the other hand, there is limited theoretical or computational literature on the evolution of the semicoherent precipitate beyond the critical size (especially with respect to the formation of the second loop). One important work in this regard is the work of Quek et

*Received 17 Sep., 2012 (No. 12-0376) [DOI: 10.1299/jmmp.7.135]

Copyright $\odot 2013$ by JSME 
al. [14], where they have considered the formation of multiple dislocation loops using dislocation dynamics. However, they have not computed the critical size for the formation of the loops.

The current work aims at the following tasks: (i) determination of the precipitate size beyond which two symmetrically positioned interfacial dislocations loops are energetically feasible as compared to the coherent state (designated as $\mathrm{r}^{* *}$ ); (ii) determine the range of positions along the interface for which the energy of the system with two loops is lower than the coherent state; (iii) compute the size of the precipitate at which a system with two loops has a lower energy as compared to that with a single loop (the size of the precipitate beyond which this occurs is designated as $r_{2}^{* *}$ ). Stable and unstable equilibrium positions of the loops will also be identified. The phrase 'symmetrically positioned' with respect to the position of the loops, is explained in the next section.

\section{Finite element methodology}

A schematic diagram of the finite element model, along with the boundary conditions, is shown in Fig. 1. The domain size $(400 \mathrm{~b} \times 400 \mathrm{~b})$ is chosen such that it simulates precipitation in an infinite matrix. The point ' $\mathrm{O}$ ' in the figure represents the origin. Mirror boundary condition (imposed as constraint on the nodes along the $y$-direction) along $\mathrm{y}=0$ line (represented as triangles with rollers) along with axisymmetry along $\mathrm{x}=0$ line (dash-dot line) generates the full domain. The surfaces at $y=400 \mathrm{~b}$ and on $\mathrm{x}=400 \mathrm{~b}$ are free-surfaces without any boundary conditions or displacement constraints.

Isotropic material properties are assumed in the current work. The material properties for the precipitate and matrix are [15]: (i) lattice parameters: a (ppt) $=a_{p}=3.56 \AA$, $\mathrm{a}$ (matrix) $=\mathrm{a}_{\mathrm{m}}=3.61 \AA$; (ii) slip system: $<110>\{111\}$; (iii) $\mathrm{b}=\sqrt{2} \mathrm{a}_{0} / 2=2.519 \AA$; (iv) $\mathrm{G}(\mathrm{ppt})=88.57 \mathrm{GPa}, \mathrm{G}$ (matrix) $=55.3 \mathrm{GPa} ;(\mathrm{v}) \vee(\mathrm{ppt})=0.2737, v$ (matrix) $=0.3234$. The properties of the precipitate and the matrix alloys are calculated through linear interpolation of the properties of the constituent elements. The elastic properties of the isotropic materials are computed by Voigt averaging the single crystal elastic constants $\left(\mathrm{C}_{\mathrm{ij}}\right)$.

The stress state of a system consisting of a spherical precipitate of $\gamma$-Fe in a matrix of a solid solution of $\mathrm{Cu}-2 \mathrm{wt} . \% \mathrm{Fe}$, is simulated by imposing eigenstrains [16,17] corresponding to the lattice misfit strain, between the precipitate and the matrix. In the current work, it is assumed that there is no $\mathrm{Cu}$ content in the $\gamma \mathrm{Fe}$ precipitate, as prescribed by Easterling and Miekk-Oja [18]. The eigenstrains are imposed as thermal strains in the numerical model. This is achieved by using a finite value of thermal expansion coefficient $(\alpha)$ in material property of the elements corresponding to the precipitate and increasing the temperature of the system $(\Delta \mathrm{T})$. The thermal expansion introduced is isotropic. The eigenstrains generated on heating $\varepsilon_{i j}^{*}$ is given by: $\varepsilon_{i j}^{*}=\delta_{i j} \alpha \Delta T$, where $\delta_{i j}$ is the Kronecker delta.

The value of the misfit strain $\left(f_{m}\right)$ can be calculated as: $\left(a_{p}-a_{m}\right) / a_{p}=-0.0136$ and is imposed in region-P in Fig. 1. The radius of the precipitate is marked as $r_{p}$ in the figure.

An interfacial misfit dislocation loop is simulated by feeding stress-free strains in region-D of the domain (Fig. 1). The value of this strain can be computed as:

$$
\varepsilon_{T}=\frac{\left(a_{[110]}+b\right)-a_{[110]}}{\left(a_{[110]}+b\right)}=\frac{1}{2}
$$

where, $a_{[110]}$ is the lattice parameter along the [110] direction, the value of which is equal to ' $b$ '. The strain imposed has a component of the eigen-strain tensor in the y-direction only (i.e. $\left.\varepsilon_{\mathrm{yy}}\right)$. The procedure of introduction of this strain is similar to that for the precipitate. This strain corresponds to the insertion of a disc of atoms and simulates a circular dislocation loop. The mirror boundary condition along $y=0$ implies the presence of another misfit dislocation loop at a distance of ' $y_{1}$ ' along $-y$ axis (i.e. this results in two symmetrically positioned dislocation loops with a separation of ' $2 y_{1}$ '). The position of the 
loop can be varied by changing the value of $y_{1}$.

The structure and energy of the core of the dislocation loop is neglected in the current work. The core radius is considered to equal to ' $5 b$ ' in most cases [19] and the core energy is usually $10 \%$ of the total strain energy [20,21]. The finite element model is implemented using the Abaqus/standard (Version 6.81) FEM software. The $400 \mathrm{~b} \times 400 \mathrm{~b}$ domain is meshed with $\mathrm{b} \times \mathrm{b}$ 4-noded quadrilateral elements.

The stress state of the precipitates of increasing size is simulated by increasing the radius of the region corresponding to the precipitate $\left(r_{p}\right)$ in Fig. 1. The strain energy of: (i) the coherent system (with a given value of $r_{p}$ ) and (ii) the system with two dislocation loops, is computed using the finite element model. The energy of the system for different positions of the interfacial loop is determined from the simulations by changing the value of $\mathrm{y}_{1}$. The critical size $r^{* *}$ is determined from the plot of strain energy versus $y_{1}$, for increasing values of $r_{p}$. A similar procedure is adopted to determine the value of $r_{2}^{* *}$.

It is assumed that the shape of the precipitate remains spherical during the entire process. The above methodology can be extended to find the critical sizes $\left(r^{* *} \& r_{2}^{* *}\right)$ for asymmetrical positions of the dislocation loops. The simulation of the system with one interfacial misfit dislocation loop along the equatorial plane has been done by imposing eigenstrain, which corresponds to that for a single dislocation (i.e. considering $\mathrm{y}_{1}=0$ ).

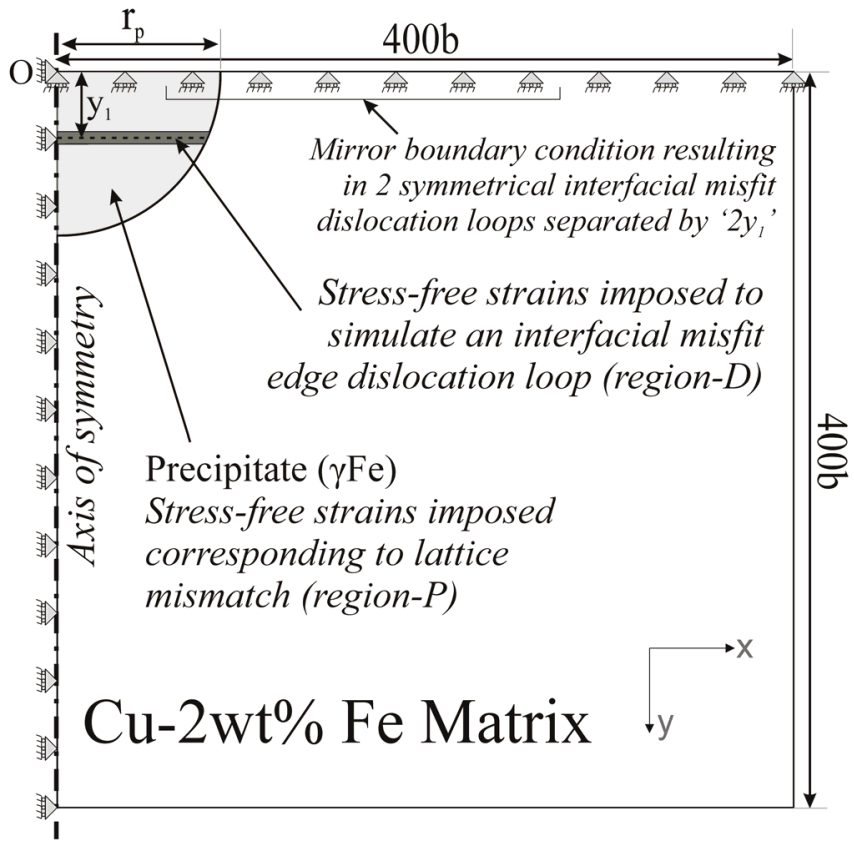

Fig. 1 Schematic of the finite element model, along with boundary conditions, used for the simulation of a coherent precipitate and two interfacial misfit dislocations. The dash-dot line is the line of axisymmetry. The boundary condition along $\mathrm{y}=0$, implies a symmetrically positioned dislocation loop at $\mathrm{y}=-\mathrm{y}_{1}$. The origin of the axis is at 'O'.

\section{Results and discussions}

The stress state of the system (plot of $\sigma_{\mathrm{yy}}$ stress contours) in the presence of two dislocation loops at a mutual distance of $40 \mathrm{~b}\left(\mathrm{y}_{1}=20 \mathrm{~b}\right)$ is shown in Fig. 2 (the figure shows a symmetrical half of the domain). The radius of the precipitate in the figure is $50 \mathrm{~b}$, which is just above the critical size $\left(r^{*}\right)$, at which a single interfacial loop becomes energetically favourable along the equatorial plane. The modified state of stress in the presence of dislocation loops is to be noted. The tensile stress state of the precipitate is partially relaxed by the compressive stresses of the dislocation loops.

In the next of set of results to be considered, three parameters are plotted in the figures: (i) $E_{c}$ (energy of the coherent precipitate), (ii) $E_{1 d}$ (energy of the system with one interfacial 
misfit dislocation loop along the equatorial plane), (iii) $\mathrm{E}_{2 \mathrm{~d}}$ (energy of the system with two dislocation loops). It should be noted that these energies are positive, while their differences could be negative (e.g $E_{1 \mathrm{~d}}-\mathrm{E}_{\mathrm{c}}$ ) depending on the position of the dislocation along the interface $\left(y_{1}\right)$. Further, unlike the procedure for determination of $r^{*}$, where $r_{p}$ is the variable along the $\mathrm{x}$-axis of the plots; in the current investigation $r_{p}$ is kept constant, while $y_{1}$ is varied (the $\mathrm{x}$-axes of the plots).

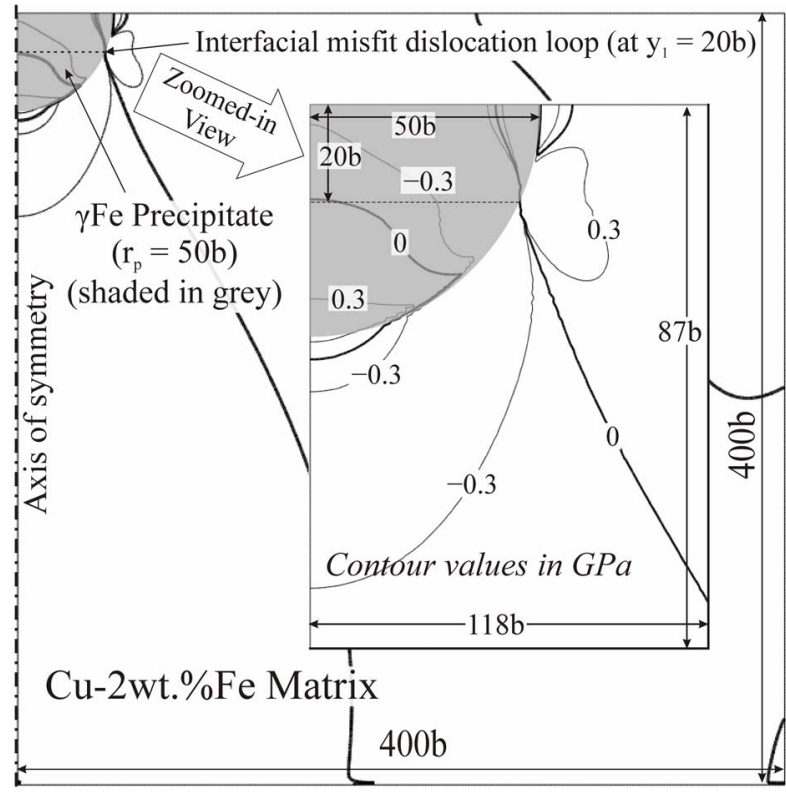

Fig. 2 Plot of $\sigma_{y y}$ contours of the precipitate in the presence of two dislocation loops separated by a distance of $40 \mathrm{~b}\left(\mathrm{y}_{1}=20 \mathrm{~b}, \mathrm{r}_{\mathrm{p}}=50 \mathrm{~b}\right)$. Inset shows the zoomed-in view of the region close to the precipitate. The

shaded region shows the precipitate in the undeformed domain.

Fig. 3 shows the variation in the energy of the system, as a function of the position of the dislocation loop $\left(\mathrm{y}_{1}\right)$, for two sizes of the precipitates $\left(\mathrm{r}_{\mathrm{p}}=50 \mathrm{~b}, 58 \mathrm{~b}\right)$. Energy of half the domain is plotted in this and subsequent figures (i.e. the total energy of the system can be obtained by doubling the value plotted).

For $r_{p}=50 b$, it is seen that: $E_{2 d}>E_{c}>E_{1 d}$ (Fig. 3a). This implies that the system with two dislocations is not favourable (for any value of $y_{1}$ ). At $r_{p}=58 b$, the value of $E_{2 d}=E_{c}$ at $\mathrm{y}_{1}=30 \mathrm{~b}$ and for other values of $\mathrm{y}_{1}: \mathrm{E}_{2 \mathrm{~d}}>\mathrm{E}_{\mathrm{c}}$ (Fig. 3b). Hence, $\mathrm{r}^{* *}=58 \mathrm{~b}$.

At $r_{p}=50 b$, the coherency stresses (and corresponding energy of the system) is not sufficient to support two dislocation loops and the repulsive interaction between the loop dominates the energy of the system, leading to a monotonic decrease in the energy as the loops are positioned at larger separations (increasing $\left.\mathrm{y}_{1}\right)$. It is to be noted that the curve $\left(\mathrm{E}_{2 \mathrm{~d}}\right.$ in Fig. 3a) has no extrema.

At $r_{p}=58 b$, the curve $\left(E_{2 d}\right.$ in Fig. $\left.3 b\right)$ has one maximum and one minimum, implying one unstable equilibrium position (at $\mathrm{y}_{1}=50 \mathrm{~b}$ ) and one stable equilibrium position (at $\mathrm{y}_{1}=$ 30b). As evident from the figure the stable position corresponds to a 'local equilibrium' and is not energetically preferred over the coherent state. However, it should be noted if loops are somehow trapped in the local minimum (e.g. preexisting dislocations in the sample forms interfacial loops), they will be stable in the local minimum. The shape of the curve $\left(E_{2 d}\right.$ in Fig. 3b) can be understood as follows. For small $y_{1}$, the repulsive interaction between the loops dominates the energy and hence there is a decrease in the energy of the system with increasing $\mathrm{y}_{1}$. For larger $\mathrm{y}_{1}$, the strain relief provided by the loops is lesser (due to smaller interaction of the dislocation stress fields with the coherency stresses, i.e. domination of the self energy of the loops), thus leading to an increase in energy with $\mathrm{y}_{1}$. Between the two regimes, the minimum in energy occurs. For $\mathrm{y}_{1}>50 \mathrm{~b}$, the loop is unstable. 


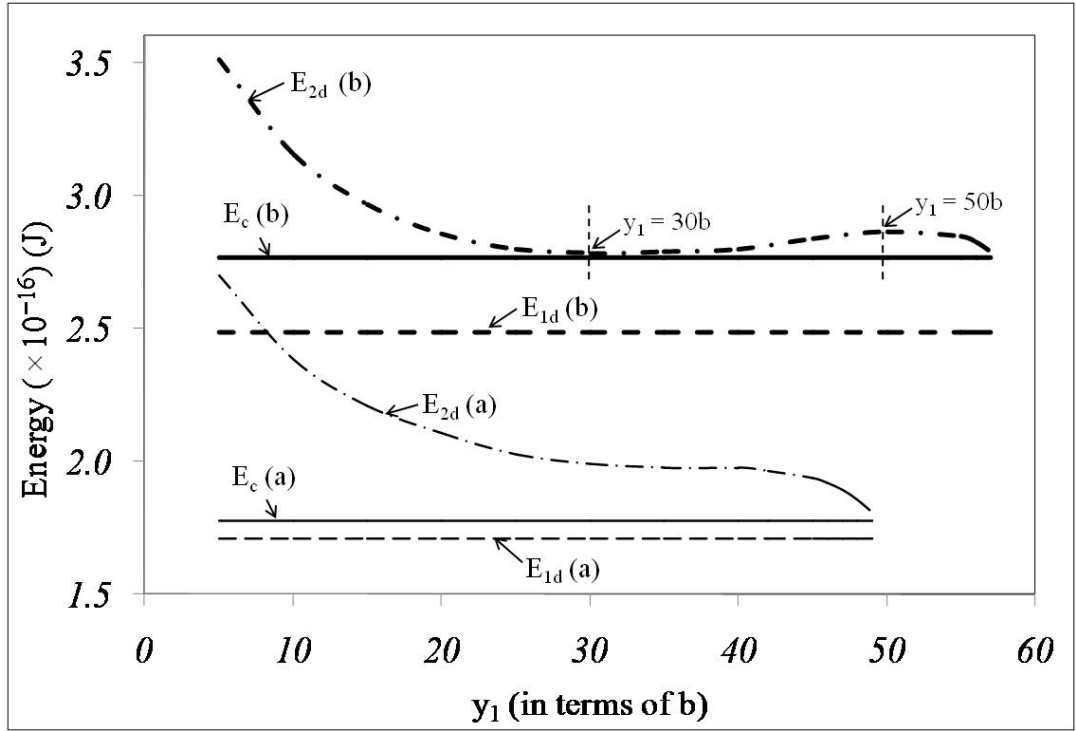

Fig. 3 The variation of the energy of the system $\left(E_{2 d}\right)$ with the position of the dislocation loop $\left(y_{1}\right)$, for: (a) $r_{p}=50 b$ (shown with thin lines), (b) $r_{p}=58 b$ (shown with thick lines). The energy of the coherent precipitate $\left(\mathrm{E}_{\mathrm{c}}\right)$ and the system with one interfacial dislocation loop $\left(\mathrm{E}_{1 \mathrm{~d}}\right)$ are included for comparison.

On growth beyond $r^{* *}$ the system with two dislocations can be energetically favored over the coherent state for a certain range of $y_{1}$ as shown in Fig. 4 (for $r_{p}=60 b, 76 b$ ). For $\mathrm{r}_{\mathrm{p}}=60 \mathrm{~b}, \mathrm{E}_{2 \mathrm{~d}}<\mathrm{E}_{\mathrm{c}}\left(\right.$ for $\mathrm{y}_{1} \in[20 \mathrm{~b}, 45 \mathrm{~b}]$ ) and $\mathrm{E}_{2 \mathrm{~d}}>\mathrm{E}_{1 \mathrm{~d}}$. This implies that the configuration with two dislocation loops is energetically favourable over the coherent state for $20 \mathrm{~b}<\mathrm{y}_{1}<$ $45 \mathrm{~b}$; however, for no symmetrical positions of the two loops, this state is preferred over the state with a single dislocation loop. The stable position is at $\mathrm{y}_{1}=35 \mathrm{~b}$ (with $\mathrm{E}_{2 \mathrm{~d}}<\mathrm{E}_{\mathrm{c}}$ ). The unstable position is at $\mathrm{y}_{1}=56 \mathrm{~b}$ and for this position $\mathrm{E}_{2 \mathrm{~d}}>\mathrm{E}_{\mathrm{c}}$.

For $r_{p}=76 b, E_{2 d}=E_{1 d}\left(\right.$ at $\left.y_{1}=30 b\right)$. This value of $r_{p}$ represents a third critical size $\left(\mathrm{r}_{2}^{* *}\right)$ : the size at which the systems with one and two dislocations have the same energy (for one position with $\mathrm{y}_{1}=30 \mathrm{~b}$ ). The plot of $\mathrm{E}_{2 \mathrm{~d}}$ with $\mathrm{y}_{1}$ shows one stable position at $\mathrm{y}_{1}=$ $30 \mathrm{~b}$ and one unstable position at $\mathrm{y}_{1}=73 \mathrm{~b}$ (Fig. $4 \mathrm{~b}$ ).

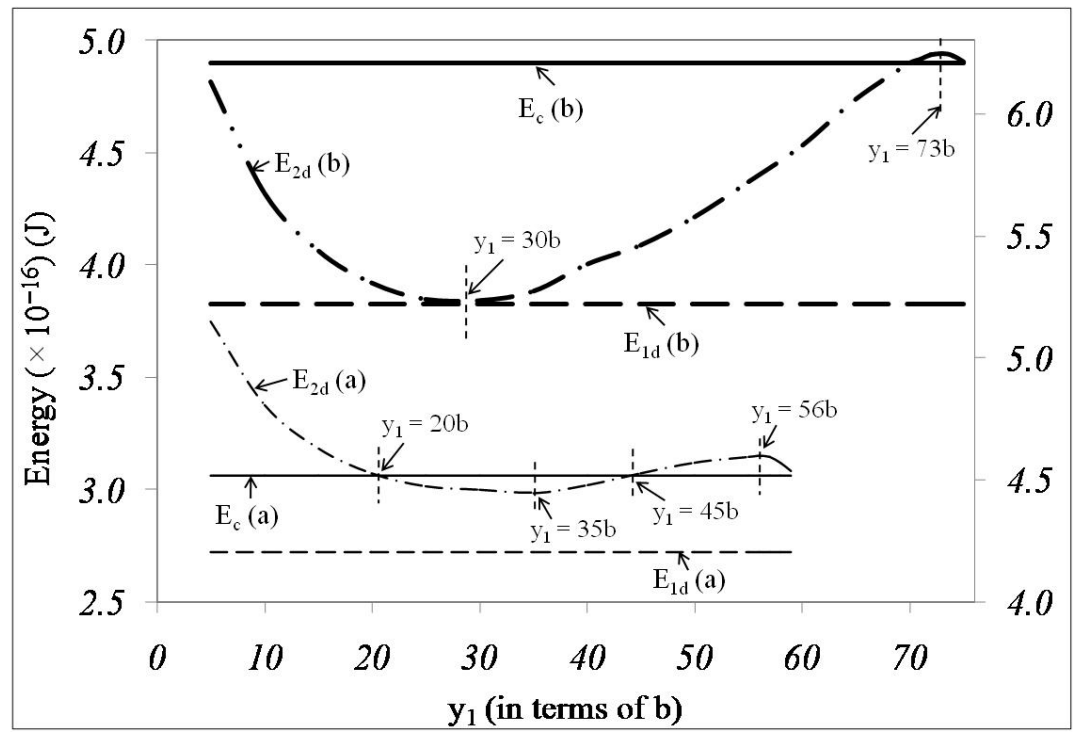

Fig. 4 The variation of the energy of the system with the position of the dislocation loop $\left(\mathrm{y}_{1}\right)$, for: (a) $r_{p}=60 b$ (shown with thin lines, primary y-axis), (b) $r_{p}=76 b$ (shown with thick lines, secondary y-axis).

On further growth, the system with two loops becomes energetically preferred over the system with single loop, for certain range of positions ( $\left.y_{1}\right)$ (as shown in Fig. 5 for $r_{p}=90 b$ ). 
For $\mathrm{r}_{\mathrm{p}}=90 \mathrm{~b}, \mathrm{E}_{2 \mathrm{~d}}<\mathrm{E}_{\mathrm{c}}$ and $\mathrm{E}_{2 \mathrm{~d}}<\mathrm{E}_{1 \mathrm{~d}}$ (for $\mathrm{y} \in[11 \mathrm{~b}, 50 \mathrm{~b}]$ ). For this radius of precipitate, there exists one stable position at $\mathrm{y}_{1}=30 \mathrm{~b}$ (where $\mathrm{E}_{2 \mathrm{~d}}<\mathrm{E}_{1 \mathrm{~d}}$ ) and one very weak unstable position at $\mathrm{y}_{1}=88 \mathrm{~b}\left(\mathrm{E}_{2 \mathrm{~d}}<\mathrm{E}_{\mathrm{c}}\right.$ and $\left.\mathrm{E}_{2 \mathrm{~d}}>\mathrm{E}_{1 \mathrm{~d}}\right)$. The results and analyses are summarized in Table 1.

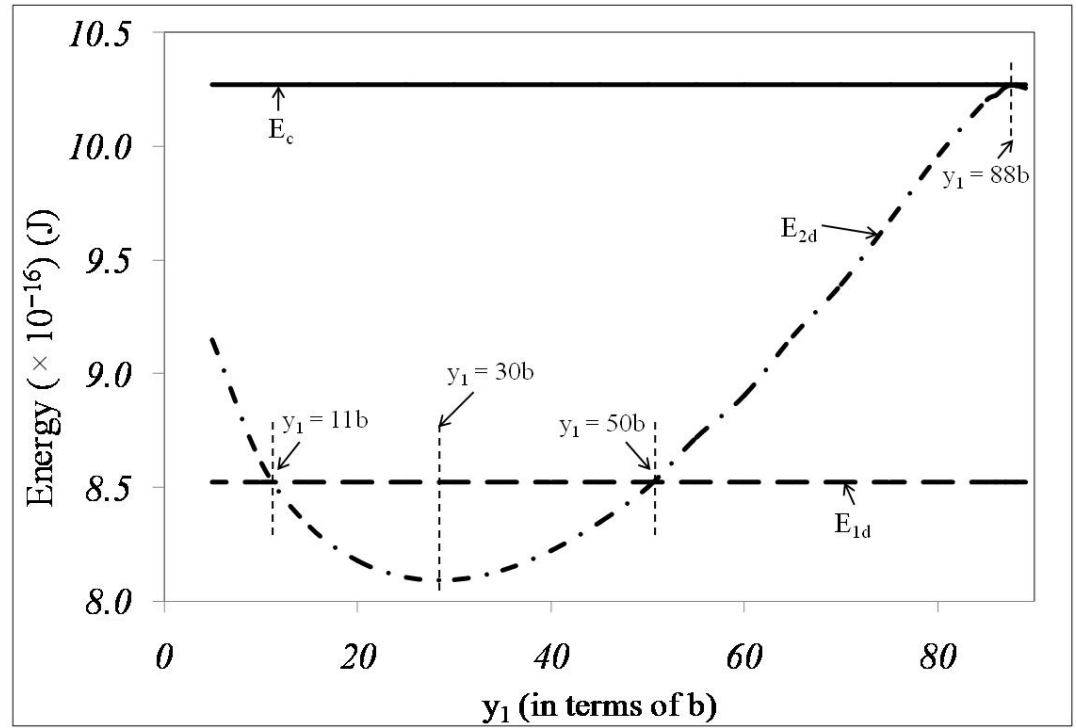

Fig. 5 The variation of the energy of the system with the position of the dislocation loop $\left(y_{1}\right)$, for $r_{p}=90 b$.

Table 1. Comparison of the relative magnitudes of the energies $\left(E_{c}, E_{1 d}, E_{2 d}\right)$ for different values of $r_{p}$.

\begin{tabular}{|c|c|c|}
\hline$r_{p}$ & Relative energies & Comments \\
\hline $47 \mathrm{~b}$ & $\begin{array}{l}E_{c}=E_{1 d} \\
E_{2 d}>E_{c}\end{array}$ & value of $\mathrm{r}^{*}$ \\
\hline$<58 b$ & $\mathrm{E}_{2 \mathrm{~d}}>\mathrm{E}_{\mathrm{c}}>\mathrm{E}_{1 \mathrm{~d}}$ & \\
\hline $58 b$ & $\begin{array}{l}E_{c}=E_{2 d} \text { for } y_{1}=30 b \\
E_{c}>E_{1 d}\end{array}$ & $\begin{array}{l}\text { value of } \mathrm{r}^{* *} \\
\text { Stable equilibrium position at } \mathrm{y}_{1}=30 \mathrm{~b} \\
\text { Unstable equilibrium position at } \mathrm{y}_{1}=50 \mathrm{~b}\end{array}$ \\
\hline$(58 b, 76 b)$ & $\begin{array}{l}E_{2 d}>E_{1 d} \\
E_{2 d}<E_{c} \text { for a range of } y_{1}\end{array}$ & $\begin{array}{l}\text { E.g. for } r_{p}=60 b, \\
E_{2 d}<E_{c} \text { for } y_{1} \in[20 b, 45 b] \\
\text { Stable equilibrium position at } y_{1}=35 b \\
\text { Unstable equilibrium position at } y_{1}=56 b\end{array}$ \\
\hline $76 b$ & $E_{2 d}=E_{1 d}$ for $y_{1}=30 b$ & value of $r_{2}^{* *}$ \\
\hline $76 b$ & $\begin{array}{l}E_{2 d}<E_{c} \\
E_{2 d}<E_{1 d} \text { for a range of } y_{1}\end{array}$ & $\begin{array}{l}\text { E.g. for } r_{p}=90 b, \\
E_{2 d}<E_{1 d} \text { for } y_{1} \in[11 b, 50 b] \\
\text { Stable equilibrium position at } y_{1}=30 b \\
\text { Unstable equilibrium position at } y_{1}=88 b\end{array}$ \\
\hline
\end{tabular}

\section{Summary and conclusions}

Finite element method has been used to simulate the stress state of a coherent precipitate and a semi-coherent precipitate with two symmetrically positioned dislocation loops. The critical size $\left(\mathrm{r}^{* *}\right)$, for the energetic feasibility of two dislocation loops, is determined by comparing the energy of the system, with and without dislocation loops. At $\mathrm{r}^{* *}$, the energy of the system with two dislocation loops is equal to that of the coherent state, for just one position of the dislocation loop. The range of positions over which the loops are energetically preferred over the coherent state is determined from the simulations (for precipitate sizes greater than $\mathrm{r}^{* *}$ ). It is seen that for a certain range of precipitate sizes, 
although the system with two dislocations is preferred over the coherent state; it is still not preferred energetically over the state with a single dislocation loop.

Even before $\mathrm{r}^{* *}$ is exceeded, there exists a local equilibrium position for the dislocation loops (i.e. if the loops somehow forms at this position, they will be stable). Positions of stable and unstable equilibrium (for the dislocation loops) are determined, for specific sizes of the precipitate exceeding $\mathrm{r}^{* *}$. The size of the precipitate after which the system with two dislocations becomes energetically equal to the system with a single dislocation (for specific positions of the loops) is determined (designated as $r_{2}^{* *}$ ). On further growth of the precipitate, the state with two dislocations becomes energetically favoured over the one with a single dislocation (for a certain range of positions of the two dislocation loops).

\section{References}

[1] D.A. Porter and K.E. Easterling, Phase Transformations in Metals and Alloys, Chapman \& Hall, London, 1992.

[2] L.M. Brown, G.R. Woolhouse and U. Valdrè, Radiation-induced coherency loss in a Cu-Co alloy, Philos. Mag., Vol. 17, Issue 148, 1968, pp.781.

[3] L.M. Brown, G.R. Woolhouse, The loss of coherency of precipitates and the generation of dislocations, Philos. Mag., Vol. 21, 1970, pp.329.

[4] G.R. Woolhouse and M. Ipohorski, On the interaction between radiation damage and coherent precipitates, Proc. R. Soc. A, Vol. 324, 1971, pp.415.

[5] W.A. Jesser, On the theory of loss of coherency by spherical precipitates, Philos. Mag., Vol. 19, 1969, pp.993.

[6] H. Brooks, Metal Interfaces (American Society for Metals), 1952, pp.20.

[7] F.J. Humphreys, The Interaction of Dislocations with Coherent Cobalt Particles in a Copper Matrix, Acta Metall., Vol. 16, 1968, pp.1069.

[8] D. Watanabe, C. Watanabe and R. Monzen , J. Mater. Sci., Vol. 43, 2008, pp.3946.

[9] G.C. Weatherly, Loss of coherency of growing particles by the prismatic punching of dislocation loops, Philos. Mag., Vol. 17, Issue 148, 1968, pp.791.

[10] S. Sen, R. Balasubramaniam and R. Sethuraman, Finite element evaluation of elasto-plastic accommodation energies during solid state transformations: Coherent, spherical precipitate in finite matrix, Scripta Metall. Mater., Vol. 33, 1995, pp.527.

[11] N. Miyano, K. Ameyama, N. Hirano and Y. Takao, FEM analysis based on the 3D-NCS model for precipitate morphology in the BCC/HCP system, Mater. Sci. Eng. A, Vol. 480, 2008, pp.464.

[12] U. Stigh, A finite element study of threading dislocations, Mech. Mater., Vol. 14, 1993, pp.179.

[13] K. Sasaki, M. Kishida and Y. Ekida, Stress analysis in continuous media with an edge dislocation by finite element dislocation model, Intl. J. Num. Meth. Engg., Vol. 54, 2002, pp.671.

[14] S.S. Quek, Y. Xiang and D.J. Srolovitz, Loss of interface coherency around a misfitting spherical inclusion, Acta Mater., Vol. 59, 2011, pp.5398.

[15] E.A. Brandes (Ed), Smithells Metals Reference Book, Butterworths, London, 1983.

[16] T. Mura, Micromechanics of Defects in Solids, Martinus Nijhoff Publishers, The Hague, 1982.

[17] R.D. Cook, D.S. Malkus, M.F. Plesha and R.J. Witt, Concepts and Application to Finite Element Analysis, John Wiley \& Sons Pvt. Ltd., Singapore, 2003.

[18] K.E. Easterling and H.M. Miekk-Oja, The Martensitic Transformation of iron precipitates in a copper matrix , Acta Metall., Vol. 15, 1967, pp.1133.

[19] G.S. Was, Fundamentals of Radiation Materials Science, Springer, New York, 2007.

[20] J.P. Hirth and J. Lothe, Theory of Dislocations, McGraw-Hill, New York, 1968.

[21] W. Bollmann, Crystal Defects and Crystalline Interfaces, Springer-Verlag, Berlin, 1970. 